\title{
Increased Apo A-I and Apo A-II Fractional Catabolic Rate in Patients with Low High Density Lipoprotein-Cholesterol Levels with or without Hypertriglyceridemia
}

Eliot A. Brinton, Shlomo Eisenberg," and Jan L. Breslow

The Rockefeller University, New York, New York 10021; and *Hadassah University Hospital, Jerusalem, Israel

\begin{abstract}
Low HDL-cholesterol (HDL-C) levels may elevate atherosclerosis risk, and often associate with hypertriglyceridemia (HTG); however, the metabolic causes of low HDL-C levels with or without HTG are poorly understood. We studied the turnover of radioiodinated HDL apolipoproteins, apo A-I and apo A-II, in 15 human subjects with low HDL-C, six with normal plasma TG levels (group 1) and nine with high TG (group 2), and compared them to 13 control subjects with normal HDL-C and TG levels (group 3). The fractional catabolic rate (FCR) was equally elevated in groups 1 and 2 vs. group 3 for both apo $A-I(0.313 \pm 0.052$ and $0.323 \pm 0.063$ vs. $0.245 \pm 0.043$ pools $/ \mathrm{d}, P=0.003)$ and apo A-II $(0.213 \pm 0.036$ and $0.239 \pm 0.037$ vs. $0.185 \pm 0.031$ pools $/ \mathrm{d}, P=0.006)$. Thus, high FCR characterized low HDL-C regardless of the presence or absence of HTG. In contrast, transport rate (TR) of apo A-I did not differ significantly among the groups and the apo A-II TR differed only between groups 2 and $3(2.15 \pm 0.57,2.50 \pm 0.39$, and $1.83 \pm 0.48 \mathrm{mg} / \mathrm{kg}$ per $d$ for groups 1 to 3 , respectively, $P$ $=0.016$ ). Several HDL-related factors were similar in groups 1 and 2 but differed in group 3 , as with FCR, including the ratio of lipoprotein lipase to hepatic lipase activity (LPL/HL) in postheparin plasma, the ratio of the HDL-C to apo A-I plus apo A-II levels, and the percent of tracer in the $d>1.21$ fraction. In linear regression analysis HDL-C levels correlated inversely with the FCR of apo A-I and apo A-II $(r=-0.74, P<0.0001$ for both). Major correlates of FCR were HDL-C/apo A-I + apo A-II, LPL/HL, and plasma TG levels.

We hypothesize that lipase activity and plasma TG affect HDL composition which modulates FCR, which in turn regulates HDL-C. Thus, HTG is only one of several factors which may contribute to elevated FCR and low HDL-C. Given the relationship of altered HDL composition with high FCR and low HDL-C levels, factors affecting HDL composition may increase atherosclerosis susceptibility. (J. Clin. Invest. 1991. 87:536-544.) Key words: high density lipoprotein • apolipoproteins A-I and A-II turnover or metabolism - hypertriglyceridemia
\end{abstract}

\section{Introduction}

Considerable epidemiologic evidence suggests that low plasma levels of high-density lipoprotein cholesterol (HDL-C) ${ }^{1}$ are

Address correspondence to Dr. Eliot A. Brinton, The Rockefeller University Hospital, Box H29, 1230 York Ave., New York, NY 10021.

Received for publication 26 December 1989 and in revised form 21 September 1990

1. Abbreviations used in this paper: FCR, fractional catabolic rate; HDL-C, high-density lipoprotein cholesterol; HL, hepatic lipase, HTG, hypertriglyceridemia; LPL, lipoprotein lipase; TG, triglyceride; TR, transport rate.

J. Clin. Invest.

(c) The American Society for Clinical Investigation, Inc.

0021-9738/91/02/0536/09 \$2.00

Volume 87, February 1991, 536-544 linked to elevated risk of atherosclerosis $(1,2)$; however, the metabolic mechanisms underlying a low HDL-C are unclear. Previous HDL turnover studies of patients with low HDL-C levels and hypertriglyceridemia (HTG) have shown an elevated fractional catabolic rate (FCR) of the major HDL apolipoproteins apo A-I (3) and apo A-II $(3,4)$. It was concluded that HTG itself was the cause of the low HDL-C level and the increase in HDL apolipoprotein FCR, yet these studies lacked the crucial comparison group of patients with low HDL-C and normal TG levels. Only one HDL turnover study has directly compared low HDL-C patients with normal or high TG (5). In that study, the low-HDL-C, normal TG subjects had normal apo A-I FCR but low TR, whereas the low-HDL-C, HTG subjects had elevated apo A-I FCR and TR. This implied a striking contrast in the metabolism of apo A-I between low-HDL-C patients with or without HTG. Because the comparison groups were small, however, and there was relatively little control over the subjects' diets during the study, we have chosen to reexamine this important issue.

In the current report, we have studied the turnover of apo A-I and apo A-II on a standard $42 \%$ fat diet under metabolic ward conditions in 15 subjects with low-HDL-C and normal or high-plasma TG compared to 13 control subjects with normal HDL-C and TG levels. We find that elevated HDL apolipoprotein FCR is the major metabolic mechanism of low HDL-C levels in both normal-TG and HTG subjects, suggesting that HTG is not a unique cause of the elevated FCR. Rather, we find that HTG is one of several factors that combine, possibly in an additive manner, to account for the increased FCR in patients with low HDL-C levels.

\section{Methods}

Subjects. 12 male and 16 female subjects were recruited for HDL metabolic studies from three sources: $(a)$ patients from the clinic of the Laboratory of Biochemical Genetics and Metabolism; $(b)$ healthy, adult volunteers working on the staff at Rockefeller University or at neighboring institutions; $(c)$ undergraduate students in a work-study program. The subjects were classified into three groups by age and sex-corrected percentiles of fasting HDL-C and TG levels (6) from values obtained on the study diet. Group 1 had HDL-C below the 20th percentile and TG below the 90th percentile and consisted of three females and three males. Group 2 had HDL-C below the 20th percentile and TG at or above the 90th percentile, and consisted of five females and four males. Group 3, the normal control group, had an HDL-C level from the 20th to the 79th percentile and a TG level below the 90th percentile, and consisted of eight females and five males. All subjects were free from hepatic, renal, thyroid, and immunologic disorders by history and laboratory screening. 14 of the 15 low-HDL-C subjects and two of the control subjects are presented for the first time in this report. The remaining control subjects were reported in recent publications from this laboratory $(7,8)$. Also, subject No. 6 (group 1) was reported in our recent study of dietary effects on HDL metabolism (8).

Subject No. 2 (group 1) had been taking an oral hypoglycemic, glyburide, $5 \mathrm{mg}$ daily for a few years at the time of the study. None of the other subjects were taking medications known to alter glucose or lipid levels. The clinical aspects of these studies were approved by the 
Institutional Review Board of the Rockefeller University, and informed consent was obtained from the subjects in all cases.

Apolipoprotein preparation and labeling. Apolipoproteins A-I and A-II were prepared from healthy donor plasma by ultracentrifugation, delipidation, and column chromatography using standard methods as previously described (7). The purified apo A-I and apo A-II were radioiodinated by the iodine monochloride method and tested for pyrogenicity and sterility as outlined recently (7).

Kinetic studies. The subjects were admitted to the Rockefeller University Hospital inpatient ward and kept on a natural food metabolic diet for $4 \mathrm{wk}$. The diet had a caloric distribution of $42 \%$ fat, $43 \%$ carbohydrate, and $15 \%$ protein. The fat content corresponded to the 50th percentile of previous American intake according to the Lipid Research Clinics study (9), and to the 75th percentile of the current American consumption according to the NHANES II survey (10). The diet had a polyunsaturated-to-saturated fat $(\mathrm{P} / \mathrm{S})$ ratio of 0.1 and contained $215 \mathrm{mg}$ cholesterol per 1,000 calories. Caloric need was estimated by the Harris-Benedict (11) equation, with adjustment for physical activity (12), and caloric intake was not altered during the study. After the first $2 \mathrm{~d}$ body weight remained constant. No alcohol intake was allowed and subjects were instructed to maintain their usual level of physical activity.

After 2 wk of equilibration on the metabolic diet, the subjects received radioiodinated apo A-I and apo A-II (10-25 $\mu \mathrm{Ci}$ of ${ }^{125} \mathrm{I}$ and 25 $\mu \mathrm{Ci}$ of ${ }^{131} \mathrm{I}$, respectively, or vice versa) by intravenous bolus injection. Subject No. 13 only received $25 \mu \mathrm{Ci}$ of ${ }^{125} \mathrm{I}$ apo A-I. Previous studies in two separate laboratories have demonstrated that radiolabeling of purified HDL apolipoproteins gives results similar to that obtained by protein labeling of intact $\operatorname{HDL}(13,14)$. This similarity is due to the rapid association of free HDL apolipoproteins with plasma HDL in vivo. To allow for thorough mixing of the tracers with HDL in the plasma compartment, the first blood sample was taken after $10 \mathrm{~min}$. Blood was then taken at $4,12,24$, and $36 \mathrm{~h}$ and then daily until $14 \mathrm{~d}$. All except the 4-, 12-, and 36-h samples were taken after an overnight fast of $12 \mathrm{~h}$. Each sample was handled and counted as outlined previously (7). Because all subjects were in steady state with regard to apo A-I and apo A-Il levels, the radioactive decay curve was plotted directly from the die-away of plasma counts. The FCR was calculated from this curve by the Matthews method (15). Absolute transport (production or synthetic) rate (TR) was calculated by multiplying the FCR by the plasma pool (apolipoprotein level times plasma volume) and dividing by the body weight. Plasma volume was determined by isotope dilution at the 0 -min point as extrapolated backwards log-linearly from the tracer clearance at earliest plasma sampling time points.

To show that the iodinated apo A-I and apo A-II associated with $\mathrm{HDL}$, in each study extra aliquots of plasma were taken $10 \mathrm{~min}$ and 7 and $14 \mathrm{~d}$ after injection. Three fractions were prepared by ultracentrifugation, $d<1.063,1.063<d<1.21$, and $d>1.21$. The density distribution of radiolabel was calculated as a percentage of total counts, and the results for the 10-min 7- and 14-d samples were averaged. The rapid equilibration of the tracer with the intravascular pools of unlabeled apolipoprotein $(13,14)$ allows the use of the density distribution of tracer as a measure of the density distribution of the subjects' own apolipoproteins.

Lipid, lipoprotein and apolipoprotein determinations. On days 1, 3, 7,10 , and 14 of the turnover period, plasma was obtained after a 12-h overnight fast for measurement of lipid, lipoprotein, and apolipoprotein levels. Lipid and lipoprotein measurements were done on fresh specimens by enzymatic methods as previously described (7). Aliquots of plasma were also stored at $-70^{\circ} \mathrm{C}$ for subsequent apolipoprotein determinations. Apo A-I levels were measured by a sandwich ELISA using a polyclonal goat antibody to human apo A-I (generously supplied by Dr. Peter Herbert) as recently reported (7). Apo A-II levels were kindly determined by Dr. John J. Albers in the Northwest Lipid Research Clinics laboratories by a radioimmunoassay based on a radial immunodiffusion assay (16). Over the 2-wk turnover period, no temporal trends were observed in the lipid, lipoprotein, and apolipoprotein levels and the mean of all five determinations was used in the data analysis.
Postheparin lipase activities. After $11 \mathrm{~d}$ on the test diet and $3 \mathrm{~d}$ before isotope injection, a postheparin lipase test was performed. Blood was drawn $15 \mathrm{~min}$ after an intravenous bolus dose of heparin, $60 \mathrm{U} / \mathrm{kg}$ body weight, and the plasma was stored at $-70^{\circ} \mathrm{C}$ until assay. Lipase activity was measured with ${ }^{3} \mathrm{H}$-triolein in a sonicated Triton $\times 100$ emulsion in the presence or absence of rabbit anti-human lipoprotein lipase (LPL) serum as reported previously (7). Lipase activity was expressed as micromoles FFA liberated per milliliter plasma per hour. LPL activity was the difference between total activity and that remaining after antibody inhibition, whereas the latter was the hepatic lipase (HL) activity.

Statistical analysis. Linear regression analysis was performed using the least squares method, and statistical significance was defined as a $P$ value $<0.05$. Differences among the groups were evaluated by analysis of variance (one-way ANOVA, Neumann-Keuls' method) using the same $P$ value cutoff. Calculations were performed on The Rockefeller University Hospital CLINFO system.

\section{Results}

The sex, age, body mass index, and lipoprotein profiles (including age and sex percentiles for HDL-C and TG) were determined for each low-HDL-C subject (Table I). The mean and standard deviation of these variables were calculated for lowHDL-C subjects with normal TG (group 1) and HTG (group 2 ), and were compared by ANOVA to that of a group of subjects with normal HDL-C and TG levels (group 3) who were studied under identical conditions $(7,8)$. Because all but two of the normal-HDL-C, normal-TG subjects (group 3) have been reported previously, only the mean values for this group are given. Mean age did not differ among the groups. Variation among groups in body mass index was confined to two obese subjects (Nos. 1 and 9). Total cholesterol and LDL-C did not differ among the groups. As expected, VLDL-C was higher in the HTG subjects of group 2 than in the normal-TG subjects of groups 1 and $3(117 \pm 62$ vs. $46 \pm 31$ and $28 \pm 14 \mathrm{mg} / \mathrm{dl}$, respectively, $P<0.0001)$. By selection, the mean HDL-C levels were lower in groups 1 and 2 compared with group 3 (35 \pm 4 and $33 \pm 6$ vs. $53 \pm 9 \mathrm{mg} / \mathrm{dl}, P<0.0001)$ with corresponding differences in HDL-C percentile $(9 \pm 3$ and $10 \pm 7$ vs. $57 \pm 17 \%, P$ $<0.0001$ ). Also by selection, the mean TG level of group 2 $(557 \pm 392 \mathrm{mg} / \mathrm{dl})$ and its percentile $(98 \pm 2 \%)$ were much higher than values of groups 1 and 3 (levels, $128 \pm 32$ and $95 \pm 31 \mathrm{mg} /$ dl, $P<0.0001$; percentiles, $60 \pm 12$ vs. $59 \pm 15 \%$, respectively, $P$ $<0.0001$ ), which did not differ significantly from each other.

The tracer distribution volumes, apolipoprotein A-I and A-II levels, and their metabolic parameters were determined (Table II) as explained in Methods. Although the decreases in mean apo A-I level of groups 1 and 2 compared to group 3 appeared to be of unequal magnitude $(106 \pm 14$ and $121 \pm 13$ vs. $138 \pm 19 \mathrm{mg} / \mathrm{dl}$, decreased 23 and $12 \%$, respectively) they were both significantly lower than group $3(P=0.0014$ for overall difference) and they did not differ significantly from each other. The FCR of apo A-I was equally elevated in groups 1 and 2 compared to group $3(0.313 \pm 0.052$ and $0.323 \pm 0.063$ vs. $0.245 \pm 0.043$ pools $/ \mathrm{d}, 28$ and $32 \%$ higher, respectively, $P$ $=0.003$ ). These relationships in overall apo A-I FCR were reflected in the plasma clearance curves of the apo A-I tracer among the members of the three subject groups. This is demonstrated by the curves for the three individual subjects with an FCR closest to the mean for their respective groups (Fig. $1 a$ ). The apo A-I TR did not differ significantly among the groups $(12.5 \pm 1.4,12.8 \pm 0.9$, and $11.5 \pm 1.9 \mathrm{mg} / \mathrm{kg}$ per $\mathrm{d}$, for groups $1-3$, respectively, $P=0.15)$. The apo A-II parameters were in 


\begin{tabular}{|c|c|c|c|c|c|c|c|c|c|c|c|}
\hline Group & Subject & Sex & Age & BMI* & TC & VLDL-C & LDL-C & HDL-C & $\underset{\text { percentile }}{\text { HDL }}$ & TG & $\begin{array}{c}\text { TG } \\
\text { percentile }\end{array}$ \\
\hline & & & $y r$ & & $m g / d l$ & $m g / d l$ & $m g / d l$ & $m g / d l$ & & $m g / d l$ & \\
\hline \multirow{8}{*}{$\begin{array}{l}\text { 1. Low HDL-C, } \\
\text { nl TG }\end{array}$} & 1 & $\mathbf{F}$ & 47 & 42.5 & 203 & 19 & 144 & 40 & 13 & 87 & 46 \\
\hline & 2 & $\mathbf{F}$ & 57 & 29.5 & 257 & 50 & 168 & 38 & 7 & 139 & 65 \\
\hline & 3 & $\mathbf{F}$ & 67 & 25.7 & 250 & 38 & 175 & 36 & 8 & 147 & 66 \\
\hline & 4 & $\mathbf{M}$ & 46 & 29.5 & 411 & 105 & 274 & 33 & 9 & 175 & 78 \\
\hline & 5 & $\mathbf{M}$ & 38 & 25.0 & 391 & 31 & 385 & 32 & 12 & 112 & 51 \\
\hline & 6 & $\mathbf{M}$ & 34 & 27.6 & 145 & 85 & 85 & 30 & 7 & 105 & 52 \\
\hline & Mean & & 48 & 30.0 & 276 & 46 & 196 & 35 & 9 & 128 & 60 \\
\hline & SD & & 12 & 6.4 & 105 & 31 & 89 & 4 & 3 & 32 & 12 \\
\hline \multirow{11}{*}{$\begin{array}{l}\text { 2. Low HDL-C, } \\
\text { HTG }\end{array}$} & 7 & $F$ & 31 & 21.3 & 197 & 46 & 109 & 43 & 18 & 138 & 95 \\
\hline & 8 & $\mathbf{F}$ & 66 & 26.5 & 311 & 79 & 195 & 37 & 9 & 282 & 97 \\
\hline & 9 & $F$ & 52 & 39.9 & 475 & 229 & 211 & 36 & 5 & 783 & 99 \\
\hline & 10 & $F$ & 41 & 21.3 & 254 & 60 & 160 & 34 & 5 & 208 & 98 \\
\hline & 11 & $\mathbf{F}$ & 41 & 25.1 & 287 & 140 & 120 & 28 & 2 & 733 & 99 \\
\hline & 12 & $\mathbf{M}$ & 48 & 32.8 & 274 & 129 & 110 & 35 & 18 & 777 & 99 \\
\hline & 13 & $\mathbf{M}$ & 45 & 25.2 & 196 & 60 & 102 & 35 & 16 & 260 & 94 \\
\hline & 14 & $\mathbf{M}$ & 37 & 25.1 & 353 & 127 & 194 & 31 & 11 & 479 & 99 \\
\hline & 15 & $\mathbf{M}$ & 58 & 27.6 & 264 & 187 & 56 & 22 & 2 & 1354 & 99 \\
\hline & Mean & & 47 & 27.2 & 290 & 117 & 140 & 33 & 10 & 557 & 98 \\
\hline & SD & & 11 & 5.9 & 85 & 62 & 53 & 6 & 7 & 393 & 2 \\
\hline \multirow{2}{*}{$\begin{array}{l}\text { 3. Nl HDL-C, } \\
\text { nl TG }\end{array}$} & Mean & & 34 & 23.0 & 233 & 28 & 152 & 53 & 57 & 95 & 59 \\
\hline & SD & & 20 & 3.0 & 128 & 14 & 120 & 9 & 17 & 31 & 15 \\
\hline Group comparison ${ }^{\ddagger}$ & & & $1=2=3$ & $1=2,2=3$ & $1=2=3$ & $1=3$ & $1=2=3$ & $1=2$ & $1=2$ & $1=3$ & $1=3$ \\
\hline$P$ value & & & NS & 0.018 & NS & $<0.0001$ & NS & $<0.0001$ & $<0.0001$ & $<0.0001$ & $<0.0001$ \\
\hline
\end{tabular}

* Abbreviations and terms: BMI $\left(\mathrm{kg} / \mathrm{m}^{2}\right)$, body mass index; TC, total cholesterol; $\mathrm{nl}$, normal; NS $(P>0.05)$, not significant. The percentile is given for the subjects' age and sex from the Lipid Research Clinic Population Study. ${ }^{\ddagger}$ Groups not differing by Neuman Keuls' ANOVA $(P>0.05)$ are separated by an equal sign (e.g., $1=2)$. For $0.001<P<0.05$ the exact $P$ value is given.

partial contrast to those of apo A-I. Apo A-II levels did not differ significantly among the three groups $(26.9 \pm 3.0$, $32.8 \pm 5.6$, and $29.6 \pm 4.8 \mathrm{mg} / \mathrm{dl}, P=0.09$ ). The mean apo A-II FCR did not differ significantly between groups 1 and 2 $(0.213 \pm 0.036$ vs. $0.239 \pm 0.037$ pools $/ \mathrm{d}$, respectively); however, compared to group $3(0.185 \pm 0.031$ pools $/ \mathrm{d})$, group 2 was significantly higher ( $29 \%$ higher, $P=0.006)$, whereas group 1 was not significantly so ( $15 \%$ higher, $P>0.1$ ). This group comparison in overall apo A-II FCR was reflected in the plasma clearance curves of apo A-II tracer in the individual subjects as typified by the three subjects with FCR closest to the group mean (Fig. $1 \mathrm{~b}$ ). The TR of apo A-II varied in that it was $37 \%$ higher in group 2 than in group $3(2.57 \pm 0.42$ vs. $1.88 \pm 0.49$ $\mathrm{mg} / \mathrm{kg}$ per d, $P<0.05$ overall), whereas group $1(2.20 \pm 0.59$ $\mathrm{mg} / \mathrm{kg}$ per d) did not differ significantly from either group 2 or 3 .

Parameters related to HDL-C levels and metabolism were also measured (Table III). Activity of the endothelial lipases, LPL, and HL were measured in postheparin plasma. Mean LPL activity in group 2 was $29 \%$ lower than group 3 (10.4 \pm 2.1 vs. $14.7 \pm 2.4 \mu \mathrm{mol} / \mathrm{ml}$ per $\mathrm{h}, P=0.001)$ but group $1(12.8 \pm 2.2$ $\mu \mathrm{mol} / \mathrm{ml}$ per $\mathrm{h}$ ) did not differ significantly from either group 2 or 3 . In contrast, there was no significant difference in $\mathrm{HL}$ among the groups $(22.1 \pm 5.1,18.4 \pm 5.0$, and $15.4 \pm 7.9 \mu \mathrm{mol} / \mathrm{ml}$ per $\mathrm{h}$ for groups 1 to 3 , respectively, $P>0.1$ ). Because LPL and $\mathrm{HL}$ are reported to have opposing effects on HDL-C levels (17, and see reference 18 for an earlier review), we calculated the LPL/HL ratio. An identical 50\% lowering of LPL/HL was seen in both groups 1 and 2 compared to group $3(0.62 \pm 0.21$ and $0.62 \pm 0.28$ vs. $1.25 \pm 0.72, P=0.018$ ). An index of HDL composition was obtained by calculating the HDL-C/apo A-I + apo A-II molar ratio from plasma levels. All three groups differed significantly from each other, with group 1 being $18 \%$ lower (16.8 \pm 0.6$)$ and group 2 being $32 \%$ lower $(13.9 \pm 2.7)$ than group $3(20.4 \pm 2.5, P<0.0001)$. The percent of each tracer found in the $d<1.063,1.063<d<1.21$ (HDL), and $d>1.21 \mathrm{~g} / \mathrm{ml}$ fractions of plasma was determined. Because the percent tracer in the $d<1.063$ fraction was very small $(<2 \%)$ and varied little, the tracer distribution in either the HDL or $d>1.21$ fraction would indicate the relative distribution between the two. Tracer distribution within the $d>1.21$ fraction was predominantly within the $1.21<d<1.25$ (VHDL) portion in several representative subjects. We, therefore, used the percent of the tracers in the $d>1.21$ fraction as an indicator of the amount of lipid poor VHDL, relative to more lipid-rich HDL. The percent of apo A-I tracer in the $d>1.21$ fraction was elevated in groups 1 and 2 compared to the normals $(20.6 \pm 7.0$ and $22.1 \pm 8.8$ vs. $11.9 \pm 3.0,73$, and $86 \%$ higher, respectively; $P$ $=0.0016)$. The percent of apo A-II tracer in $d>1.21$ fraction was less in every subject than for the apo A-I tracer; however, the relationship among the three subject groups were similar for both apolipoproteins. Groups 1 and 2 had significantly 
Table II. Plasma Levels, FCR and TR of Apo A-I and Apo A-II

\begin{tabular}{|c|c|c|c|c|c|c|c|}
\hline \multirow[b]{2}{*}{ Group } & \multirow[b]{2}{*}{ Subject } & \multicolumn{3}{|c|}{ Apo A-I } & \multicolumn{3}{|c|}{ Apo A-II } \\
\hline & & Level & FCR & TR & Level & FCR & TR \\
\hline \multirow{9}{*}{$\begin{array}{l}\text { 1. Low HDL-C } \\
\text { nl TG }\end{array}$} & & $m g / d l$ & pools/d & $m g / k g / d$ & $m g / d l$ & pools/d & $m g / k g / d$ \\
\hline & 1 & 128 & 0.322 & 13.2 & 27.1 & 0.186 & 1.62 \\
\hline & 2 & 111 & 0.399 & 12.9 & 31.9 & 0.247 & 2.28 \\
\hline & 3 & 111 & 0.249 & 12.6 & 28.1 & 0.193 & 2.47 \\
\hline & 4 & 104 & 0.320 & 12.0 & 25.9 & 0.207 & 1.93 \\
\hline & 5 & 92 & 0.273 & 9.5 & 22.5 & 0.178 & 1.53 \\
\hline & 6 & 89 & 0.316 & 12.5 & 25.7 & 0.268 & 3.05 \\
\hline & Mean & 106 & 0.313 & 12.1 & 26.9 & 0.213 & 2.15 \\
\hline & SD & 14 & 0.052 & 1.4 & 3.0 & 0.036 & 0.57 \\
\hline \multirow{11}{*}{$\begin{array}{l}\text { 2. Low HDL-C, } \\
\text { HTG }\end{array}$} & 7 & 138 & 0.234 & 10.9 & 35.1 & 0.178 & 2.11 \\
\hline & 8 & 117 & 0.304 & 12.9 & 32.4 & 0.195 & 2.28 \\
\hline & 9 & 139 & 0.328 & 12.6 & 43.0 & 0.267 & 3.19 \\
\hline & 10 & 113 & 0.415 & 12.6 & 28.8 & 0.255 & 1.97 \\
\hline & 11 & 128 & 0.377 & 12.1 & 38.9 & 0.246 & 2.41 \\
\hline & 12 & 113 & 0.321 & 13.4 & 26.2 & 0.268 & 2.60 \\
\hline & 13 & 124 & 0.251 & 11.6 & 30.9 & - & - \\
\hline & 14 & 97 & 0.282 & 12.7 & 26.5 & 0.226 & 2.75 \\
\hline & 15 & 121 & 0.393 & 13.5 & 33.5 & 0.281 & 2.69 \\
\hline & Mean & 121 & 0.323 & 12.5 & 32.8 & 0.239 & 2.50 \\
\hline & SD & 13 & 0.063 & 0.8 & 5.6 & 0.037 & 0.39 \\
\hline \multirow{4}{*}{$\begin{array}{l}\text { 3. } \mathrm{nl} \text { HDL-C } \\
\text { nl TG } \\
\text { Group comparison } \\
P \text { Value }\end{array}$} & Mean & 138 & 0.245 & 11.2 & 29.6 & 0.185 & 1.83 \\
\hline & SD & 19 & 0.043 & 1.9 & 4.8 & 0.031 & 0.48 \\
\hline & & $1=2$ & $1=2$ & $1=2=3$ & $1=2=3$ & $1=2,1=3$ & $1=2,1=3$ \\
\hline & & 0.001 & 0.003 & NS & NS & 0.006 & 0.016 \\
\hline
\end{tabular}

See Table I for explanation of group comparison.

more apo A-II tracer in $d>1.21$ compared to group 3 (13.6 \pm 4.4 and $14.1 \pm 5.9$ vs. $8.2 \pm 2.3,66$, and $72 \%$ higher, respectively; $P=0.006$ ).

The primary classification of subjects was by age and sexspecific percentiles, but we repeated the group comparisons after reassigning the subjects using the absolute cutoffs of $\mathbf{4 0}$ $\mathrm{mg} / \mathrm{dl}$ for HDL-C and $200 \mathrm{mg} / \mathrm{dl}$ for TG. By these criteria, one woman (subject No. 1) was moved from group 1 to group 3, one woman (subject No. 7) moved from group 2 to group 3, and one man moved from group 3 to group 1. ANOVA of these absolute-cutoff groups produced only two minor changes from the original percentile-based analysis. The body mass index no longer differed among the groups, and the percent of the apo A-II label in the $d>1.21$ fraction for group 1 no longer differed significantly from group 3 .

To further explore the mechanisms of low HDL-C levels across the range of TG levels studied, we evaluated interrelationships among HDL-C levels, its metabolic parameters, and TG levels by linear regression analysis. HDL-C had a strong inverse correlation with the FCR of apo A-I and apo A-II ( $r$ $=-0.74$ for both, $P<0.0001$, Fig. 2), but HDL-C did not correlate with apo A-I TR $(r=0.22, P>0.1)$. Although HDL-C did correlate with apo A-II TR $(r=0.54, P=0.003)$, this correlation explains little more than half as much of the variance in HDL-C $\left(r^{2}=0.29\right)$ as does the correlation with either FCR $\left(r^{2}=0.55\right)$. As expected, HDL-C correlated inversely with TG in single linear regression $(r=-0.58, P=0.001)$; however, in stepwise multivariate linear regression TG did not add significantly to the prediction of HDL-C variability by the FCR of apo A-I or of apo A-II.

We next tested for the reported relationship between FCR and TG (Fig. 3). Univariate linear regression analysis revealed a positive correlation between apo A-I FCR and plasma TG ( $r$ $=0.53, P=0.004$ ); however, the significance of this relationship depended upon inclusion of the normal subjects $(r=0.40$, $P=0.14$ with groups 1 and 2 alone). Apo A-II FCR also correlated significantly with TG levels, and this relationship was independent of the normal subject group ( $r=0.65$ and $0.66, P$ $<0.001$ and $P=0.01$, with and without group 3 , respectively).

Given the significant differences among the three subject groups in lipase activity, the HDL/apo A-I + apo A-II ratio and density distribution of tracer, we tested for possible relationships of FCR and TR of apo A-I and apo A-II with these factors. The FCRs of apo A-I and apo A-II had inverse relationships of borderline and moderate significance, respectively, with LPL activity $(r=-0.37$ and $-0.49, P=0.060$ and 0.010 , respectively). The TRs of apo A-I and apo A-II also had inverse correlations of borderline and high significance, respectively, with LPL activity $(r=-0.36$ and $-0.62, P=0.062$ and $<0.001$, respectively). By contrast, the FCRs of both apo A-I and apo A-II correlated positively with $\mathrm{HL}$ activity $(r=0.58$ and $0.44, P=0.002$ and 0.026 , respectively) but neither the TR of apo A-I nor of apo A-II correlated with HL activity ( $r$ $=-0.08$ and 0.14 , respectively). The FCRs of apo A-I and apo 

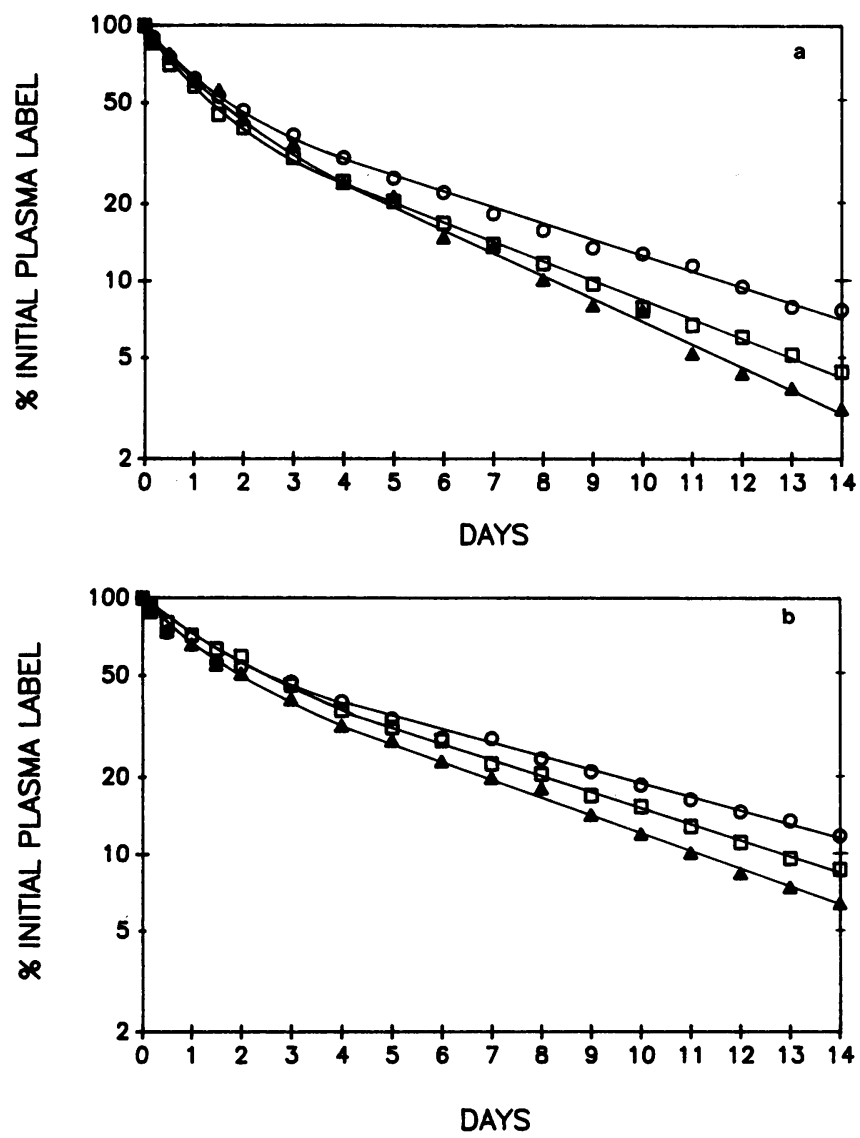

Figure 1. Plasma clearance curves for the member of each subject group with the overall FCR closest to his group mean. Low-HDL-C, normal TG subjects are denoted by open boxes (ㅁ), low-HDL-C HTG subjects (group 2 ) by solid triangles ( $\triangle$ ), and control subjects by open circles (o). (a) Apo A-I tracer curves: subject 6 for group 1, subject 12 for group 2, and a male subject for group 3. (b) Apo A-II tracer curves: subject 4 for group 1 , subject 11 for group 2 , and a second male subject for group 3 .

A-II correlated well with the LPL/HL ratio $(r=-0.67$ and -0.64 , respectively, $P<0.001$, Fig. 4 ), perhaps more strongly than with HL. The TRs of apo A-I and apo A-II had an absent or borderline correlation, respectively, with LPL/HL ( $r$ $=-0.11$ and $-0.36, P=0.08$ for the latter). The FCRs of apo A-I and apo A-II correlated strongly and inversely with the HDL-C/apo A-I + apo A-II ratio $(r=-0.69$ and -0.74 respectively, $P<0.0001$, Fig. 5). The TRs of apo A-I and apo A-II also had borderline and strong inverse correlations, respectively, with the HDL-C/apo A-I + apo A-II ratio $(r=-0.37$ and $-0.67, P=0.054$ and $<0.001$, respectively). The FCR, but not the TR, of apo A-I correlated with the percent of apo A-I tracer found in the $d>1.21$ fraction $(r=0.52$ and $0.21, P=0.004$ and $>0.1$, respectively). In contrast, neither the FCR nor the TR of apo A-II correlated significantly with the percent of apo A-II tracer in the $d>1.21$ fraction $(r=0.04$ and $0.27, P>0.1)$.

Due to the importance of FCR in predicting HDL-C, we explored the interrelationships of FCR with other study parameters by stepwise multivariate linear regression analysis. The HDL-C/apo A-I + apo A-II ratio was the strongest univariate predictor of apo A-I FCR $(r=-0.69, P<0.001)$, and the $\mathrm{LPL} / \mathrm{HL}$ ratio made the largest addition to this correlation (multiple $r=0.78, P<0.01$ for the step up). TG levels, as a third parameter, did not add significantly to this prediction of apo A-I FCR by HDL-C/apo A-I + apo A-II and LPL/HL. LPL/HL was a strong univariate predictor of apo A-I FCR ( $r$ $=-0.67, P<0.001$ ), and plasma TG did contribute significantly to this prediction $(r=0.76, P<0.01$ for the step up). The percent of apo A-I tracer in the $d>1.21$ fraction was also a univariate predictor of apo A-I FCR $(r=0.52, P=0.004)$ and added significantly to the prediction of apo A-I FCR by plasma TG ( $r=0.64, P<0.05$ for the step up). Tracer distribution did not, however, add to the prediction of apo A-I FCR by LPL/ HL or HDL/apo A-I + apo A-II, separately or together.

Given the importance of the HDL-C/apo A-I + apo A-II ratio as a single and multivariate predictor of apo A-I FCR, we tested for correlations of this ratio with factors expected to influence HDL composition. TG was the strongest univariate predictor of HDL-C/apo A-I + apo A-II $(r=-0.74, P<0.0001)$. The $\mathrm{LPL} / \mathrm{HL}$ ratio also was a strong univariate predictor of HDL-C/apo A-I + apo A-II $(r=0.58, P=0.002)$ and LPL/HL added strikingly to the prediction of that ratio by TG $(r=0.84$, $P<0.01$ for the step up). The percent apo A-I tracer in $d>1.21$ also correlated with the HDL-C/apo A-I + apo A-II ratio ( $r$ $=-0.45, P=0.02$ ) but it did not add significantly to the prediction of this ratio by TG or LPL/HL separately or together in multivariate analysis.

Finally, we compared the female and male subjects from the low HDL-C groups. Age, BMI, and the lipid and lipoprotein values did not differ. The women had higher HDL-C levels, as expected ( $37 \pm 5 \mathrm{vs.} 31 \pm 5 \mathrm{mg} / \mathrm{dl}, P=0.03)$, although their HDL-C percentiles were not higher $(8.4 \pm 5.1$ vs. $10.4 \pm 5.7 \%, P$ $=0.4)$. Also, the women had higher levels of apo A-I $(123 \pm 12$ vs. $106 \pm 14 \mathrm{mg} / \mathrm{dl} P=0.02)$ and apo A-II $(33.2 \pm 5.6$ vs. 27.4 $\pm 3.6, P=0.04)$. Interestingly, despite these differences in the HDL-C, apo A-I, and apo A-II levels there were no significant differences in any of the metabolic parameters $(P=0.4-$ $0.6)$ nor in any of the other measured parameters $(P=0.2-0.9)$ except for a borderline significant reduction of apo A-II tracer in the $d>1.21$ fraction in women $(11.7 \pm 4.3$ vs. $16.8 \pm 5.0 \%$ for women vs. men, respectively, $P=0.06$ ).

\section{Discussion}

The major goal of this study was to elucidate the metabolic mechanisms of low HDL-C levels. In particular we wished to explore the role of HTG, with which low HDL-C is often, but not always, associated. Six published HDL turnover studies have included three or more subjects with low HDL-C $(<20$ th percentile for age and sex) either with or without HTG. Three of these studies examined patients with low HDL-C and HTG $(3,4,19)$. The two studies which included normal HDL-C controls $(3,4)$, both found that the low-HDL-C, HTG patients had elevated FCR, in the form of either apo A-I (3) or apo A-II (3, 4). It was presumed that HTG was the cause of the hypercatabolism but no low HDL-C normal TG comparison group was included. Two other reports studied subjects with low HDL-C and normal TG levels $(13,20)$, but neither included a HTG comparison group, and both lacked sufficient numbers either of patients or of normal HDL-C controls to draw firm conclusions regarding a metabolic mechanism.

Only one previously published study has directly addressed the question of the interaction between low HDL-C and HTG in HDL metabolism (5). Le and Ginsberg examined apo A-I turnover in seven patients with low HDL-C and normal TG, 
Table III. Postheparin Lipase Activity, HDL Composition and Density Distribution of Injected Tracers

\begin{tabular}{|c|c|c|c|c|c|c|c|}
\hline \multirow[b]{2}{*}{ Group } & \multirow[b]{2}{*}{ Subject } & \multirow[b]{2}{*}{ LPL $^{2}$} & \multirow[b]{2}{*}{ HL } & \multirow[b]{2}{*}{ LPL/HL } & \multirow{2}{*}{$\frac{\text { HDL-C }}{\text { Apo A-I + Apo A-II }}$} & \multicolumn{2}{|c|}{$\begin{array}{l}\text { Percent tracer } \\
\text { in } \mathrm{d}>1.21\end{array}$} \\
\hline & & & & & & Apo A-I & Apo A-II \\
\hline & \multicolumn{4}{|c|}{$\mu \mathrm{mol} / \mathrm{ml} / \mathrm{h}$} & $M$ & $g / m l$ & $\mathrm{~g} / \mathrm{ml}$ \\
\hline \multirow[t]{8}{*}{ 1. Low HDL-C nl TG } & 1 & 14.7 & 18.9 & 0.78 & 16.9 & 18.4 & 14.6 \\
\hline & 2 & 13.2 & 20.5 & 0.64 & 17.0 & 12.4 & 7.8 \\
\hline & 3 & 14.2 & 18.0 & 0.79 & 16.6 & 14.0 & 8.4 \\
\hline & 4 & 9.6 & 31.1 & 0.31 & 16.1 & 29.9 & 16.4 \\
\hline & 5 & 14.7 & 18.7 & 0.79 & 17.7 & 22.3 & 16.3 \\
\hline & 6 & 10.4 & 25.4 & 0.41 & 16.3 & 26.9 & 18.1 \\
\hline & Mean & 12.8 & 22.1 & 0.62 & 16.8 & 20.6 & 13.6 \\
\hline & SD & 2.2 & 5.1 & 0.21 & 0.6 & 7.0 & 4.4 \\
\hline \multirow[t]{11}{*}{ 2. Low HDL-C, HTG } & 7 & 11.1 & 8.8 & 1.26 & 15.9 & 18.4 & 11.4 \\
\hline & 8 & 9.4 & 26.6 & 0.35 & 15.9 & 10.2 & 4.7 \\
\hline & 9 & 10.2 & 16.7 & 0.61 & 12.4 & 24.1 & 15.4 \\
\hline & 10 & 8.8 & 22.6 & 0.39 & 15.3 & 33.6 & 15.3 \\
\hline & 11 & 11.8 & 16.2 & 0.73 & 10.4 & 20.5 & 15.8 \\
\hline & 12 & 10.8 & 16.2 & 0.67 & 16.2 & 25.0 & 20.3 \\
\hline & 13 & 9.8 & 17.5 & 0.56 & 14.3 & 12.9 & - \\
\hline & 14 & 7.1 & 20.0 & 0.36 & 16.1 & 36.5 & 22.0 \\
\hline & 15 & 14.4 & 20.8 & 0.69 & 8.9 & 17.8 & 7.7 \\
\hline & Mean & 10.4 & 18.4 & 0.62 & 13.9 & 22.1 & 14.1 \\
\hline & $\mathrm{SD}$ & 2.1 & 5.0 & 0.28 & 2.7 & 8.8 & 5.9 \\
\hline \multirow[t]{2}{*}{ 3. Nl HDL-C, nl TG } & Mean & 14.7 & 15.4 & 1.25 & 20.4 & 11.9 & 8.2 \\
\hline & SD & 2.4 & 7.9 & 0.72 & 2.5 & 3.0 & 2.3 \\
\hline Group comparison & & $1=3$ & $1=2=3$ & $1=2$ & $\neq$ & $1=2$ & $1=2$ \\
\hline$P$ value & & 0.001 & NS & 0.018 & $<0.0001$ & 0.002 & 0.006 \\
\hline
\end{tabular}

See Table I for explanation of group comparison. * Abbreviations: LPL, lipoprotein lipase; HL, hepatic lipase. ${ }^{\ddagger}$ All three groups differed significantly from each other.

four patients with low HDL-C and elevated TG, and four subjects with normal HDL-C and normal TG. By ANOVA, the low HDL-C, normal TG group was found to have a low production (transport) rate and a normal FCR compared to controls, whereas the HTG group was found to have high TR and FCR. Although the low TR and the lack of elevated FCR in the low-HDL-C normal TG group contrast with the current paper, when we performed linear regression analysis of the data in their report, we found at least three important parallels with our own results. Their data revealed a strong inverse correlation between HDL-C and apo A-I FCR $(r=-0.64, P=0.01)$, a strong direct correlation between TG and apo A-I FCR $(r$ $=0.64, P=0.01$ ), and the lack of a correlation between HDL-
C levels and apo A-I TR ( $r=0.1, P=\mathrm{NS})$. By this analysis, their data support our conclusion that FCR is more important than TR in determining HDL-C levels (see below). In addition, even though by ANOVA the low-HDL-C, normal TG patients did not, as a group, have an FCR above the normal group, four of the seven individuals with low HDL-C and normal TG did have an FCR above the range of the normal subjects. The finding that the other three patients had a low TR as their sole HDL metabolic abnormality might be explained by differences in diet between their study and ours. Although the percent total fat was comparable between the studies, its degree of saturation differed substantially (P/S 0.4 vs. 0.1 ). More importantly, the previous study did not employ metabolic diet conditions. We

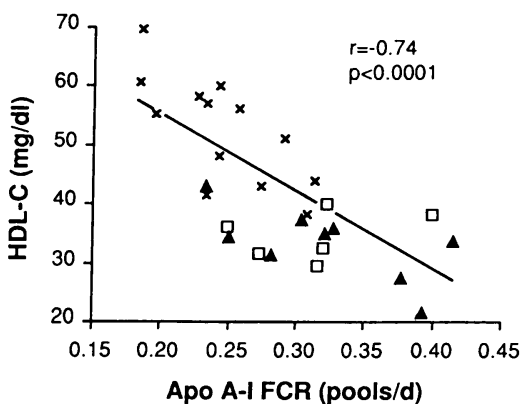

Apo A-I FCR (pools/d)

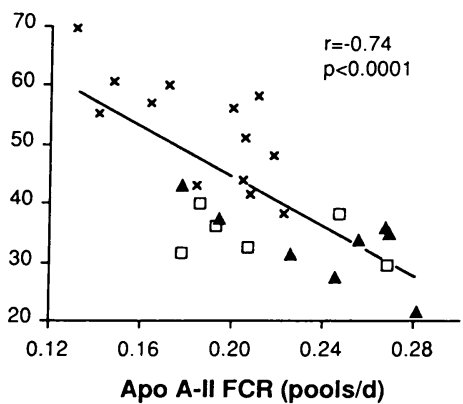

Figure 2. Relationships between HDL-C levels (milligrams per deciliter) and the FCR of apo A-I and apo A-II (pools per day). Low HDL-C, normal TG subjects (group 1) are denoted by the open boxes ( $\square$ ), low-HDL-C, HTG subjects (group 2) by solid triangles $(\triangle)$, and control subjects (group 3 ) by the symbol $X$. 

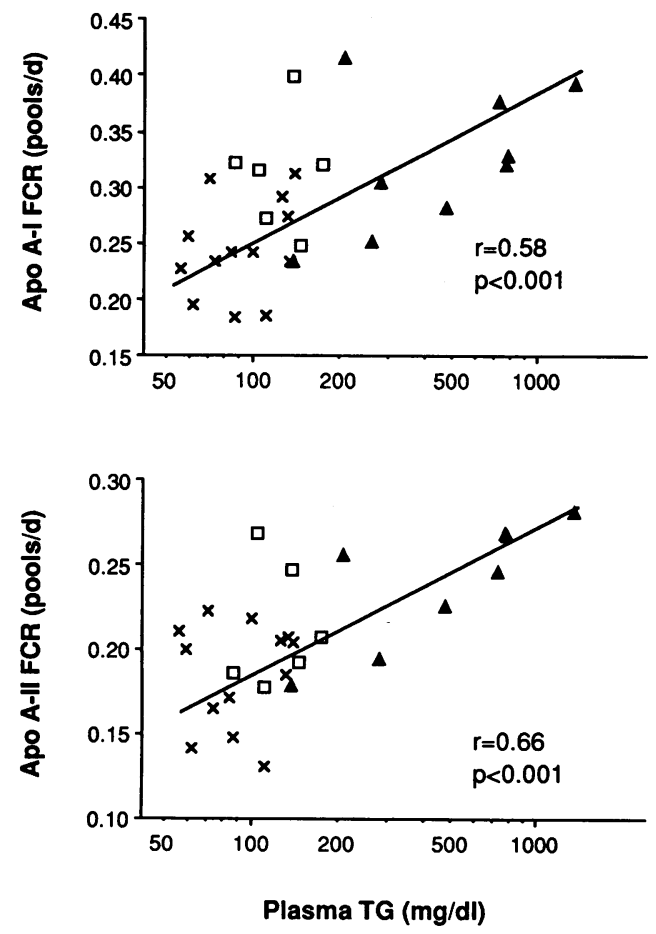

Figure 3. Relationships between the FCR of apo A-I and apo A-II (pools per day) and plasma TG levels (milligrams per deciliter). Symbols for groups 1-3 are as in Fig. 1.

have recently demonstrated that dietary changes within the range of intake commonly seen in outpatients can have profound effects on HDL-C levels and HDL apolipoprotein metabolism (8). On a very low fat intake, many of our normal subjects developed low-HDL-C and low-apo A-I TR without HTG, similar to the subjects in Le and Ginsberg's report. Given the relative lack of dietary control in their study, their subjects with low-HDL-C, normal TG and low-apo A-I TR may have had these findings inadvertently due to low fat intake. An alternate explanation is that one or more of the low HDL-C normal TG group had a truly low TR, independent of
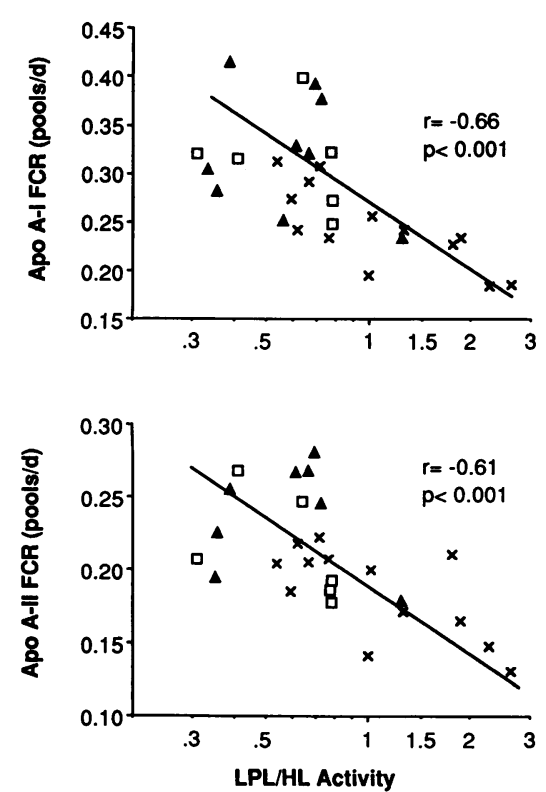

Figure 4. Relationships between the FCR of apo A-I and apo A-II (pools per day) and the LPL/HL activity ratio. Symbols for groups 1-3 are as in Fig. 1.
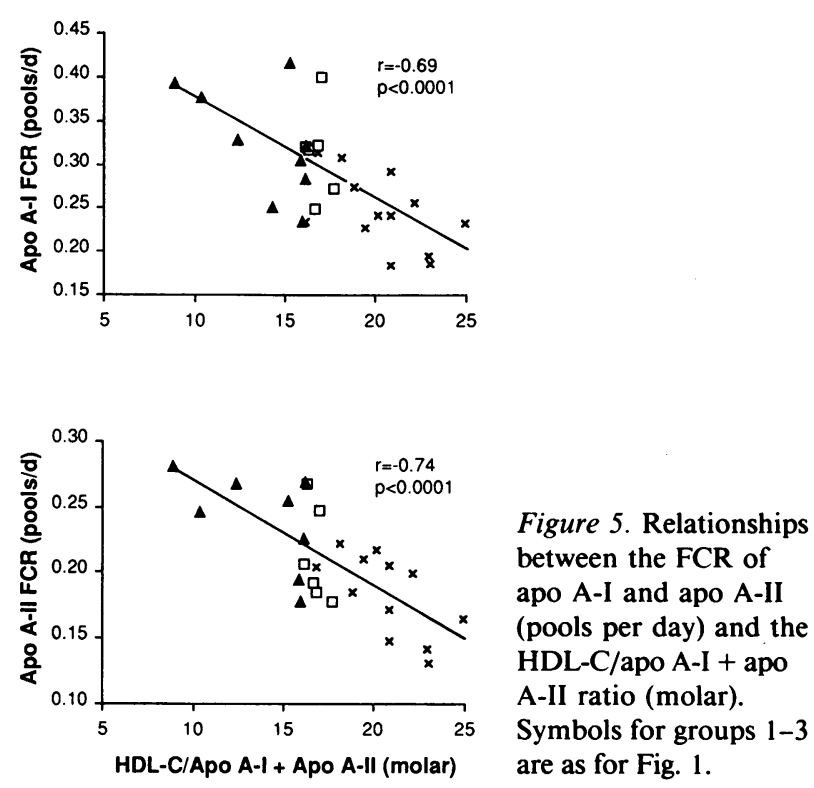

diet; however, we have been unable to find any such individuals in our metabolic studies of apparently similar patients.

Previously, men and women with normal HDL-C levels have been found to differ both in FCR and TR of HDL apolipoproteins (13). In our study, among control subjects the women had a lower FCR of apo A-I and apo A-II than the men $(P<0.05$ for both). In contrast, among our low-HDL-C subjects we found a striking similarity in HDL metabolism between the men and women. Despite having the expected higher levels of HDL-C, apo A-I, and apo A-II, these women had values indistinguishable from their male counterparts both for the metabolic parameters and for the other measured factors. The apparent paradox of unequal plasma levels but comparable metabolism is explained by the finding of lower plasma volume as a percent of body weight in the women $(3.2 \pm 0.7 \%$ for women vs. $3.8 \pm 0.6 \%$ for men). That is, production of the same amount of HDL apolipoprotein per kilogram body weight into a smaller plasma volume resulted in higher plasma levels in the women. Thus, the mechanism of gender differences in HDL-C and apo A-I levels appears to be independent of the causes of low HDL-C levels among our hypoalphalipoproteinemic subjects. Recently, a striking parallel to this finding was reported by Melchior et al. (21) in a study of cynomolgus monkeys. Among the primates, the females' higher plasma apo A-I levels were accompanied neither by lower FCR nor by higher TR on a per kilogram basis. Rather, the female monkeys had lower plasma volume per unit body weight, similar to the low-HDL-C women in our study, and this difference alone accounted for the higher plasma levels. Thus, cynomolgus monkeys share with our low HDL-C subjects a common mechanism of gender differences HDL concentration.

The metabolic parameters FCR and TR given in this study reflect only the average apo A-I and apo A-II molecule in each subject. It is, however, well established that HDL particles vary considerably in size and composition (22). Both of these factors would be expected to affect the turnover of apo A-I and apo A-II. Indeed, the current study demonstrates a strong correlation between the average composition of HDL particles and the average apolipoprotein FCR. Thus, given the compositional heterogeneity of HDL it is highly probable that HDL particles 
are metabolically heterogeneous. Due to exchange of apolipoproteins among HDL particles and continuous remodeling of HDL lipid composition (see reference 18 for review), it appears impossible to delineate this metabolic heterogeneity directly by current methods. Nevertheless, metabolic heterogeneity of apo A-I and apo A-II molecules can be inferred by complex modeling of turnover data such as the work of Zech et al. (23). Such advanced mathematical analysis has shown great promise in providing a more detailed view of the complexities of HDL metabolism, and the lack of such model detail is a major limitation of this study. Nevertheless, the role of such methods is to establish inflections in the plasma clearance curve rather than to move it significantly. Since the average FCR is simply the reciprocal of the area under the clearance curve, a more complex modeling procedure should not cause any meaningful change in the average FCR, nor in the average TR because it derives from the FCR. In fact, when Zech et al. (23) performed complex modeling on a large group of subjects analyzed previously with simple modeling by Schaefer et al. (13), the average FCR was not altered significantly for any one subject nor for one group versus another (Zech, L., personal communication). Thus, the simple modeling method used in this study probably did not greatly affect the average metabolic data given, and it is even less likely that the relationships among the study groups were altered. Furthermore, by providing just one FCR and TR value per apoprotein per subject, we facilitated the analysis of interrelationships between these metabolic parameters and the many other physiologic variables measured in this study. Our data on the average FCR of apo A-I and apo A-II reveal several striking correlations which suggest a physiologic outline of HDL metabolism.

We evaluated the relationship of HDL-C to parameters of HDL apolipoprotein metabolism and related factors in subjects with low HDL-C with or without HTG. We found hypercatabolism of apo A-I to a comparable degree in both low HDL-C groups; thus, elevated FCR of the principal HDL apolipoprotein appears to be characteristic of low HDL-C, regardless of TG levels. On the other hand, the FCR of apo A-II was elevated significantly only in the HTG patients. Nevertheless, the prediction of HDL-C levels by apo A-I or apo A-II FCR was not enhanced significantly by the addition of TG levels in multivariate linear regression. This suggests that the contribution of TG to control of HDL-C levels may be mediated via an effect on FCR. We found a strong positive relationship between FCR and TG by univariate linear regression analysis; however, the correlation between apo A-I FCR and TG depended upon the inclusion of the normal subjects. That is, looking solely within the low-HDL-C group, TG levels did not correlate with apo A-I FCR. In contrast, apo A-II FCR correlated with TG both with and without the normal subjects. Thus, TG levels may correlate more strongly or consistently with the FCR of apo A-II than with the FCR of apo A-I. This suggests that plasma TG levels may have a different effect on the catabolism of HDL particles containing apo A-I and A-II compared to those containing apo A-I but not apo A-II (22). Given the apparent importance of the FCR of apo A-I and apo A-II in determining HDL-C levels, we carefully explored potential determinants of FCR in addition to plasma TG levels. Two indices of HDL composition, the HDL-C/apo A-I + apo A-II ratio, and the percent of apo A-I tracer in the $d>1.21$ fraction, both correlated strongly with the apo A-I FCR, inversely and directly, respectively. We previously found an inverse correlation between FCR and the HDL-C/apo A-I + apo
A-II ratio in women with high HDL-C levels (7), similar to the correlation in this report. That study and this one together strongly suggest that HDL composition is an important determinant of FCR across the usual range of HDL-C levels. The single best predictor of apo A-I FCR was the HDL-C/apo A-I + apo A-II ratio. This ratio, in turn was best predicted (over $70 \%$ of the variability in multiple linear regression) by TG and LPL/HL. The LPL/HL ratio highlights reciprocal changes in LPL and HL activity. A recent population-based study found reduced LPL and increased HL activities in subjects with low HDL-C (17). Elevated HL activity has been correlated with enhanced FCR of HDL apolipoproteins $(3,7)$, and it has been suggested that this may relate to HL-mediated catabolism of HDL phospholipids and TG. Because ANOVA and linear regression of our data suggested that the LPL/HL ratio correlated more strongly with HDL-C and FCR than did HL alone, we feel that reduced LPL activity may also play a role in the increased FCR among low-HDL-C subjects. The joint effect of LPL and HL on FCR could be due to their opposing effects on HDL composition. LPL provides phospholipid for HDL enlargement, and HL readily catabolizes HDL phospholipid. Thus, a low LPL/HL ratio should promote depletion of HDL surface lipid. A coordinate role of elevated TG levels may be the promotion of removal of poorly catabolized cholesteryl ester in exchange for TG in the HDL core (see reference 18 for review). This TG is readily hydrolyzed by HL, causing depletion of HDL core lipid. Thus, we hypothesize that TG levels and the LPL/HL ratio are important determinants of HDL composition, which appears to be a major determinant of apo A-I and apo A-II FCR, which, in turn, may determine HDL-C levels (see Fig. 6). The dual contribution by TG levels and the LPL/HL ratio to HDL composition may explain an apparent paradox in our results. Although HTG contributes to low HDL-C by elevating FCR, subjects with normal TG may still have low HDL-C and rapid FCR if they have non-TG-dependent mechanisms capable of producing abnormalities in HDL composition to a degree similar to that of HTG patients.

In summary, we have demonstrated that high apo A-I and apo A-II FCR is the major metabolic mechanism of low HDL$C$, regardless of the presence or absence of HTG. Nevertheless, HTG can contribute to lowering of HDL-C levels, and this may occur via an effect of TG levels on FCR. The promotion of FCR by HTG may be additive to the effect of a low LPL/HL ratio, and both of these factors appear to be mediated through lipid depletion of HDL. Given the importance of low HDL-C levels as predictors of atherosclerosis risk, we suggest that elevated FCR of apo A-I and apo A-II may constitute an important metabolic mechanism underlying such a susceptibility. Furthermore, as potential determinants of FCR, TG levels, postheparin lipase activity, and HDL composition may also play important mechanistic roles in atherogenesis.

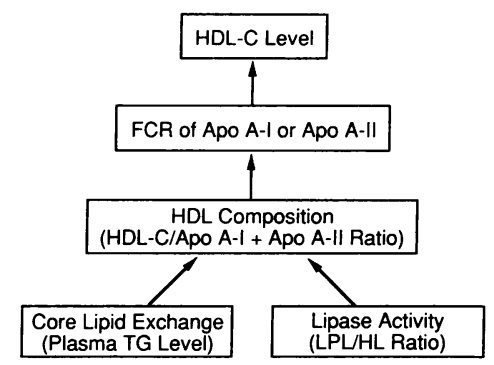

Figure 6. Hypothetical schema of the factors influencing the metabolism and levels of HDL. Determinants of HDL composition, its effect on the FCR of apo A-I and apo A-II, and the effect of FCR on HDL-C level are illustrated. 


\section{Acknowledgments}

The authors are indebted to Dr. John Albers for performing the apo A-II assays, to Dr. Rudolph Zechner for establishing the lipase assay, and to Dr. Margo Denke and Dr. Li-Shin Huang for establishing the apo A-I assay. The authors thank Dr. Nassar Khan, Ms. Michele Estilo, Ms. Margie Timmons, Ms. Katie Tsang, and Ms. Bahareh Sahami for expert technical assistance, Ms. Cindy Seidman and the dietary and nursing staff of The Rockefeller University Hospital for expert clinical assistance, and the turnover subjects for their patience and cooperation.

This work was supported in part by grants from the National Institutes of Health (HL-33714, HL-32435, HL-36461, and CA-29502), a Clinical Investigator Award (HL-02034) and a General Clinical Research Center grant (M01-RR0102), in addition to a grant from the Bankers Trust Company Group, as well as general support from the Pew Trusts. Dr. Jan L. Breslow is an Established Investigator of the American Heart Association.

\section{References}

1. Miller, G. J., and N. E. Miller. 1975. Plasma-high-density-lipoprotein concentration and development of ischaemic heart-disease. Lancet. i:16-19.

2. Miller, N. E. 1986. High density lipoprotein as a predictor of clinical coronary heart disease. In Artherosclerosis VII. N. H. Fidge and P. J. Nestel, editors. Elsevier Science Publishing, Amsterdam. 61.

3. Saku, K., P. S. Gartside, B. A. Hynd, S. G. Mendoza, and M. L. Kashyap. 1985. Apolipoprotein AI and AII metabolism in patients with primary high-density lipoprotein deficiency associated with familial hypertriglyceridemia. Metab. Clin. Exp. 34:754-764.

4. Rao, S. N., P. J. Magill, N. E. Miller, and B. Lewis. 1980. Plasma high-density lipoprotein metabolism in subjects with primary hypertriglyceridaemia: altered metabolism of apoproteins AI and AII. Clin. Sci. (Lond.). 59:359-367.

5. Le, N.-A., and H. N. Ginsberg. 1988. Heterogeneity of apolipoprotein A-I turnover in subjects with reduced concentrations of plasma high density lipoprotein cholesterol. Metab. Clin. Exp. 37:614-617.

6. Lipid Research Clinics Populations Studies Data Book. 1980. The Prevalence Study. Vol. 1. United States Department of Health and Human Services, National Institutes of Health, Bethesda, MD.

7. Brinton, E. A., S. Eisenberg, and J. L. Breslow. 1989. Elevated high density lipoprotein cholesterol levels correlate with decreased apo A-I and apo A-II fractional catabolic rate in women. J. Clin. Invest. 84:262-269.

8. Brinton, E. A., S. Eisenberg, and J. L. Breslow. 1990. A low-fat diet de- creases high density lipoprotein (HDL) cholesterol levels by decreasing $\mathrm{HDL}$ apolipoprotein transport rates. J. Clin. Invest. 85:144-151.

9. Lipid Research Clinics Population Studies Data Book. 1982. The Prevalence Study: Nutrient Intake. Vol. II. United States Department of Health and Human Services, National Institutes of Health, Bethesda, MD. 42.

10. National Health and Nutrition Examination Survey. 1983. Dietary Intake Source Data. II. National Center for Health Statistics. Office of Health Research Statistics and Technology, Hyattsville, MD. 48-52.

11. Harris, J. A., and F. G. Benedict. 1919. A Biometric Study of Basal Metabolism in Man. J. B. Lippincott Co., Philadelphia.

12. Mahalko, J. R., and L. K. Johnson. 1980. Accuracy of predictions of long-term energy needs. J. Am. Diet. Assoc. 77:557-561.

13. Shaefer, E. J., L. A. Zech, L. L. Jenkins, T. J. Bronzert, E. A. Rubalcaba, F. T. Lindgren, R. L. Aamodt, and H. B. Brewer, Jr. 1982. Human apolipoprotein A-I and A-II metabolism. J. Lipid Res. 23:850-862.

14. Malmendier, C. L., C. Delcroix, and J. P. Amcryckx. 1983. In vivo metabolism of human apoprotein A-I-phospholipid complexes: comparison with human high density lipoprotein-apoprotein A-I metabolism. Clin. Chim. Acta. 131:201210.

15. Matthews, C. M. E. 1957. The theory of tracer experiments with ${ }^{131} \mathrm{I}$-labelled plasma proteins. Phys. Med. Biol. 2:36-53.

16. Cheung, M. C., and J. J. Albers. 1977. The measurement of apolipoprotein A-I and A-II levels in men and women by immunoassay. J. Clin. Invest. 60:43-50.

17. Kuusi, T., C. Ehnholm, J. Viikari, R. Harkonen, E. Vartiainen, P. Puska, and M.-R. Taskinen. 1989. Postheparin plasma lipoprotein and hepatic lipase are determinants of hypo- and hyperalphalipoproteinemia. J. Lipid Res. 30:11171126.

18. Eisenberg, S. 1984. High density lipoprotein metabolism. J. Lipid Res. 25:1017-1058.

19. Packard, C. J., J. M. Stewart, J. L. J. C. Third, H. G. Morgan, T. D. V. Lawrie, and J. Shepherd. 1980. Effects of nicotinic acid therapy on high-density lipoprotein metabolism in type II and type IV hyperlipoproteinaemia. Biochim. Biophys. Acta. 618:53-62.

20. Blum, C. B., R. I. Levy, S. Eisenberg, M. Hall III, R. H. Goebel, and M. Berman. 1977. High density lipoprotein metabolism in man. J. Clin. Invest. 60:795-807.

21. Melchior, G. W., C. K. Castle, T. J. Vidmar, H. G. Polites, and K. R. Marrotti. 1990. Apo A-I metabolism in cynomolgus monkeys: male-female differences. Biochim. Biophys. Acta. 1043:97-105.

22. Cheung, M. C., and J. J. Albers. 1979. Distribution of cholesterol and apolipoprotein A-I and A-II in human high density lipoprotein subfractions separated by $\mathrm{CsCl}$ equilibrium gradient centrifugation: evidence for $\mathrm{HDL}$ subpopulations with differing A-I/A-II molar ratios. J. Lipid Res. 20:200-207.

23. Zech, L. A., E. J. Schaefer, T. J. Bronzert, R. L. Aamodt, and H. B. Brewer, Jr. 1983. Metabolism of human apolipoproteins A-I and A-II: compartmental models. J. Lipid Res. 24:60-71. 